\title{
Single-spin asymmetries in semi-inclusive deep-inelastic scattering on a transversely polarized hydrogen target
}

A. Airapetian, ${ }^{18}$ N. Akopov ${ }^{30}$ Z. Akopov, ${ }^{30}$ M. Amarian,,${ }^{80}$ A. Andrus, ${ }^{16}$ E.C. Aschenauer,${ }^{8}$ W. Augustyniak, ${ }^{29}$ R. Avakian ${ }^{30}$ A. Avetissian, ${ }^{30}$ E. Avetissian, ${ }^{12}$ A. Bacchetta,${ }^{23}$ P. Bailey,${ }^{16}$ D. Balin,,${ }^{21}$ M. Beckmann,${ }^{7}$ S. Belostotski, ${ }^{21}$ N. Bianchi, ${ }^{12}$ H.P. Blok,${ }^{20}{ }^{28}$ H. Böttcher, ${ }^{8}$ A. Borissov,${ }^{15}$ A. Borysenko, ${ }^{12}$ M. Bouwhuis,${ }^{16}$ A. Brüll, ${ }^{17}$ V. Bryzgalov, ${ }^{22}$ G.P. Capitani, ${ }^{12}$ M. Cappiluppi,${ }^{11}$ T. Chen,${ }^{4}$ G. Ciullo, ${ }^{11}$ M. Contalbrigo,${ }^{11}$ P.F. Dalpiaz ${ }^{11}$ R. De Leo, ${ }^{3}$ M. Demey,${ }^{20}$ L. De Nardo,${ }^{1}$ E. De Sanctis,${ }^{12}$ E. Devitsin, ${ }^{19}$ P. Di Nezza, ${ }^{12}$ M. Düren, ${ }^{14}$ M. Ehrenfried, ${ }^{10}$ A. Elalaoui-Moulay,${ }^{2}$ G. Elbakian,${ }^{30}$ F. Ellinghaus, ${ }^{8}$ U. Elschenbroich, ${ }^{13}$ R. Fabbri,${ }^{20}$ A. Fantoni, ${ }^{12}$ A. Fechtchenko, ${ }^{9}$ L. Felawka, ${ }^{26}$ S. Frullani, ${ }^{24}$ G. Gapienko, ${ }^{22}$ V. Gapienko, ${ }^{22}$ F. Garibaldi, ${ }^{24}$ K. Garrow, ${ }^{26}$ G. Gavrilov ${ }^{7}, 26$ V. Gharibyan, ${ }^{30}$ O. Grebeniouk, ${ }^{21}$ I.M. Gregor, ${ }^{8}$ C. Hadjidakis,${ }^{12}$ K. Hafidi, ${ }^{2}$ M. Hartig, ${ }^{14}$ D. Hasch, ${ }^{12}$ M. Henoch, ${ }^{10}$ W.H.A. Hesselink,${ }^{20}{ }^{28}$ A. Hillenbrand,${ }^{10}$ M. Hoek,${ }^{14}$ Y. Holler,${ }^{7}$ B. Hommez ${ }^{13}$ I. Hristova, ${ }^{8}$ G. Iarygin, ${ }^{9}$ A. Ilyichev,${ }^{7}$ A. Ivanilov, ${ }^{22}$ A. Izotov, ${ }^{21}$ H.E. Jackson, ${ }^{2}$ A. Jgoun, ${ }^{21}$ R. Kaiser, ${ }^{15}$ E. Kinney, ${ }^{6}$ A. Kisselev, ${ }^{6}$ T. Kobayashi, ${ }^{27}$ M. Kopytin, ${ }^{8}$ V. Korotkov, ${ }^{22}$ V. Kozlov, ${ }^{19}$ B. Krauss,${ }^{10}$ V.G. Krivokhijine, ${ }^{9}$ L. Lagamba, ${ }^{3}$ L. Lapikás, ${ }^{20}$ A. Laziev, ${ }^{20,}{ }^{28}$ P. Lenisa, ${ }^{11}$ P. Liebing, ${ }^{8}$ L.A. Linden-Levy, ${ }^{16}$ W. Lorenzon, ${ }^{18}$ H. Lu, ${ }^{5}$ J. Lu, ${ }^{26}$ S. Lu ${ }^{14}$ B.-Q. Ma,${ }^{4}$ B. Maiheu, ${ }^{13}$ N.C.R. Makins,${ }^{16}$ Y. Mao, ${ }^{4}$ B. Marianski, ${ }^{29}$ H. Marukyan, ${ }^{30}$ F. Masoli, ${ }^{11}$ V. Mexner, ${ }^{20}$ N. Meyners, ${ }^{7}$ T. Michler ${ }^{10}$ O. Mikloukho, ${ }^{21}$ C.A. Miller, ${ }^{1,26}$

Y. Miyachi ${ }^{27}$ V. Muccifora, ${ }^{12}$ A. Nagaitsev,${ }^{9}$ E. Nappi,${ }^{3}$ Y. Naryshkin, ${ }^{21}$ A. Nass, ${ }^{10}$ M. Negodaev,${ }^{8}$ W.-D. Nowak ${ }^{8}$ K. Oganessyan,${ }^{7}{ }^{12}$ H. Ohsuga, ${ }^{27}$ A. Osborne, ${ }^{15}$ N. Pickert,${ }^{10}$ D.H. Potterveld,${ }^{2}$ M. Raithel, ${ }^{10}$ D. Reggiani, ${ }^{11}$ P.E. Reimer, ${ }^{2}$ A. Reischl,${ }^{20}$ A.R. Reolon, ${ }^{12}$ C. Riedl,${ }^{10}$ K. Rith,,${ }^{10}$ G. Rosner, ${ }^{15}$ A. Rostomyan, ${ }^{30}$ L. Rubacek, ${ }^{14}$ J. Rubin, ${ }^{16}$ D. Ryckbosch, ${ }^{13}$ Y. Salomatin, ${ }^{22}$ I. Sanjiev,${ }^{2}{ }^{21}$ I. Savin, ${ }^{9}$ A. Schäfer, ${ }^{23}$ C. Schill, ${ }^{12}$ G. Schnell,${ }^{8,27}$ K.P. Schüler,${ }^{7}$ J. Seele,${ }^{16}$ R. Seidl, ${ }^{10}$ B. Seitz, ${ }^{14}$ R. Shanidze,${ }^{10}$ C. Shearer,${ }^{15}$

T.-A. Shibata, ${ }^{27}$ V. Shutov, ${ }^{9}$ K. Sinram, ${ }^{7}$ W. Sommer, ${ }^{14}$ M. Stancari, ${ }^{11}$ M. Statera, ${ }^{11}$ E. Steffens, ${ }^{10}$ J.J.M. Steijger, ${ }^{20}$ H. Stenzel, ${ }^{14}$ J. Stewart,${ }^{8}$ F. Stinzing, ${ }^{10}$ P. Tait,${ }^{10}$ H. Tanaka, ${ }^{27}$ S. Taroian, ${ }^{30}$ B. Tchuiko, ${ }^{22}$ A. Terkulov, ${ }^{19}$ A. Trzcinski, ${ }^{29}$ M. Tytgat,${ }^{13}$ A. Vandenbroucke, ${ }^{13}$ P.B. van der Nat,${ }^{20}$ G. van der Steenhoven, ${ }^{20}$ Y. van Haarlem, ${ }^{13}$ M.C. Vetterli, ${ }^{25,}{ }^{26}$ V. Vikhrov,${ }^{21}$ M.G. Vincter, ${ }^{1}$ C. Vogel,${ }^{10}$ J. Volmer ${ }^{8}$ S. Wang, ${ }^{5}$ J. Wendland, ${ }^{25,26}$ J. Wilbert, ${ }^{10}$ G. Ybeles Smit, ${ }^{20,}{ }^{28}$ Y. Ye ${ }^{5}$ Z. Ye, ${ }^{5}$ S. Yen, ${ }^{26}$ B. Zihlmann, ${ }^{13}$ and P. Zupranski ${ }^{29}$

(The Hermes Collaboration)

${ }^{1}$ Department of Physics, University of Alberta, Edmonton, Alberta T6G 2J1, Canada

${ }^{2}$ Physics Division, Argonne National Laboratory, Argonne, Illinois 60439-4843, USA

${ }^{3}$ Istituto Nazionale di Fisica Nucleare, Sezione di Bari, 70124 Bari, Italy

${ }^{4}$ School of Physics, Peking University, Beijing 100871, China

${ }^{5}$ Department of Modern Physics, University of Science and Technology of China, Hefei, Anhui 230026, China

${ }^{6}$ Nuclear Physics Laboratory, University of Colorado, Boulder, Colorado 80309-0446, USA

${ }^{7}$ DeSY, 22603 Hamburg, Germany

${ }^{8}$ DESY 15738 Zeuthen, Germany

${ }^{9}$ Joint Institute for Nuclear Research, 141980 Dubna, Russia

${ }^{10}$ Physikalisches Institut, Universität Erlangen-Nürnberg, 91058 Erlangen, Germany

${ }^{11}$ Istituto Nazionale di Fisica Nucleare, Sezione di Ferrara and

Dipartimento di Fisica, Università di Ferrara, 44100 Ferrara, Italy

${ }^{12}$ Istituto Nazionale di Fisica Nucleare, Laboratori Nazionali di Frascati, 00044 Frascati, Italy

${ }^{13}$ Department of Subatomic and Radiation Physics, University of Gent, 9000 Gent, Belgium

${ }^{14}$ Physikalisches Institut, Universität Gießen, 35392 Gießen, Germany

${ }^{15}$ Department of Physics and Astronomy, University of Glasgow, Glasgow G12 8QQ, United Kingdom

${ }^{16}$ Department of Physics, University of Illinois, Urbana, Illinois 61801-3080, USA

${ }^{17}$ Laboratory for Nuclear Science, Massachusetts Institute of Technology, Cambridge, Massachusetts 02139, USA

${ }^{18}$ Randall Laboratory of Physics, University of Michigan, Ann Arbor, Michigan 48109-1120, USA

${ }^{19}$ Lebedev Physical Institute, 117924 Moscow, Russia

${ }^{20}$ Nationaal Instituut voor Kernfysica en Hoge-Energiefysica (NIKHEF), 1009 DB Amsterdam, The Netherlands

${ }^{21}$ Petersburg Nuclear Physics Institute, St. Petersburg, Gatchina, 188350 Russia

${ }^{22}$ Institute for High Energy Physics, Protvino, Moscow region, 142281 Russia

${ }^{23}$ Institut für Theoretische Physik, Universität Regensburg, 93040 Regensburg, Germany

${ }^{24}$ Istituto Nazionale di Fisica Nucleare, Sezione Roma 1, Gruppo Sanità

and Physics Laboratory, Istituto Superiore di Sanità, 00161 Roma, Italy

${ }^{25}$ Department of Physics, Simon Fraser University, Burnaby, British Columbia V5A 1S6, Canada

${ }^{26}$ TRIUMF, Vancouver, British Columbia V6T 2A3, Canada

${ }^{27}$ Department of Physics, Tokyo Institute of Technology, Tokyo 152, Japan 


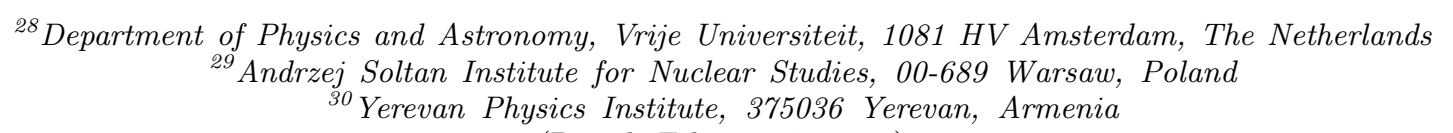

(Dated: February 7, 2008)

\begin{abstract}
Single-spin asymmetries for semi-inclusive electroproduction of charged pions in deep-inelastic scattering of positrons are measured for the first time with transverse target polarization. The asymmetry depends on the azimuthal angles of both the pion $(\phi)$ and the target spin axis $\left(\phi_{S}\right)$ about the virtual photon direction and relative to the lepton scattering plane. The extracted Fourier component $\left\langle\sin \left(\phi+\phi_{S}\right)\right\rangle_{U T}^{\pi}$ is a signal of the previously unmeasured quark transversity distribution, in conjunction with the so-called Collins fragmentation function, also unknown. The Fourier component $\left\langle\sin \left(\phi-\phi_{S}\right)\right\rangle_{U T}^{\pi}$ of the asymmetry arises from a correlation between the transverse polarization of the target nucleon and the intrinsic transverse momentum of quarks, as represented by the previously unmeasured Sivers distribution function. Evidence for both signals is observed, but the Sivers asymmetry may be affected by exclusive vector meson production.
\end{abstract}

PACS numbers: 13.60.-r, 13.88.+e, 14.20.Dh, 14.65.-q

The nucleon is a bound state containing quarks with momenta of order $\Lambda_{Q C D} \simeq 200 \mathrm{MeV}$. As the masses of the quarks of flavor $q=u p(u)$ or $\operatorname{down}(d)$ are much smaller than this, their internal motion is relativistic. The quark substructure of hadrons is often probed in the Deep-Inelastic Scattering (DIS) of leptons i.e. the absorption by a quark of a spacelike virtual photon with large squared four-momentum $q^{2}=-Q^{2}$. The essence of these experiments is captured by the Parton Model in which partons (ie. quarks and gluons) are scattered quasielastically by the lepton. The physics is most transparent in the frame in which the nucleon target moves contrary to the photon with "infinite" momentum. In this frame the transverse motion of the partons is "frozen" during the interaction time, while their transverse momenta are obviously unchanged. After averaging over this intrinsic transverse momentum $p_{T}$, three fundamental distributions in longitudinal quark momentum can be interpreted as number densities. Two of these have been experimentally explored in some detail-the unpolarized density $q(x)$ [1, 2], and the helicity density $\Delta q(x) \equiv q^{\rightrightarrows}(x)-q \stackrel{\rightleftarrows}{\rightleftarrows}(x)$ reflecting the probability of finding the helicity of the quark to be the same as that of the target nucleon [3]. Here $x=Q^{2} /(2 P \cdot q)$ is the dimensionless Bjorken scaling variable representing the momentum fraction of the target nucleon carried by the parton, where $P$ is the four-momentum of the target proton. Viewed in the same helicity basis, the third distribution known as transversity [4, 5, 6], $\delta q$ or alternatively $h_{1}^{q}$, is related to a forward scattering amplitude involving helicity flip of both quark and target nucleon $\left(N^{\Rightarrow} q^{\leftarrow} \rightarrow N^{\Leftarrow} q^{\rightarrow}\right)$ and has no probabilistic interpretation in this basis. However, it is a number density in a basis of transverse spin eigenstates: $\delta q=q^{\uparrow \Uparrow}-q^{\uparrow \Downarrow}$. The transversity and helicity densities may differ because dynamically bound light quarks move relativistically, in which regime boosts and rotations don't commute. Hence the measurement of these differences can shed light on the dynamics of nonperturbative QCD.

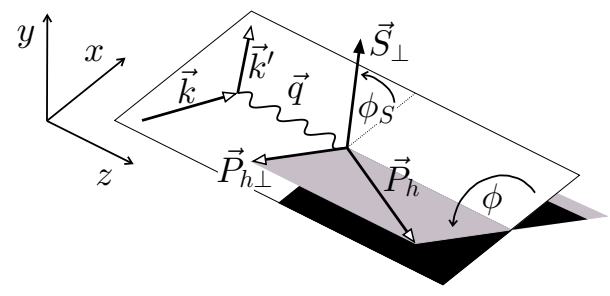

FIG. 1: The definitions of the azimuthal angles of the hadron production plane and the axis of the relevant component $\vec{S}_{\perp}$ of the target spin, relative to the plane containing the momentum $\vec{k}\left(\vec{k}^{\prime}\right)$ of the incident (scattered) lepton. Explicitly, $\phi=\frac{\vec{q} \times \vec{k} \cdot \vec{P}_{h}}{\left|\vec{q} \times \vec{k} \cdot \vec{P}_{h}\right|} \cos ^{-1} \frac{\vec{q} \times \vec{k} \cdot \vec{q} \times \vec{P}_{h}}{|\vec{q} \times \vec{k}|\left|\vec{q} \times \vec{P}_{h}\right|}$ and $\phi_{S}=$ $\frac{\vec{q} \times \vec{k} \cdot \vec{S}_{\perp}}{\left|\vec{q} \times \vec{k} \cdot \vec{S}_{\perp}\right|} \cos ^{-1} \frac{\vec{q} \times \vec{k} \cdot \vec{q} \times \vec{S}_{\perp}}{|\vec{q} \times \vec{k}|\left|\vec{q} \times \vec{S}_{\perp}\right|}$, where $0<\cos ^{-1}<\pi$.

Transversity has thus far remained unmeasured because it is chiral-odd, and hard interactions conserve chirality. However, it may be probed by a process involving some additional chiral-odd structure. If a hadron produced from the struck quark is detected in addition to the scattered lepton, the transverse polarization of the struck quark can influence the transverse momentum component $\vec{P}_{h \perp}$ of the hadron orthogonal to the virtual photon direction, and thereby influence its distribution in the azimuthal angle $\phi$ about the virtual photon direction relative to the lepton scattering plane [7] (see Fig. 1). The fragmentation function $H_{1}^{\perp}$ describing this spinmomentum correlation is indeed chiral-odd, and also odd under naive time reversal (T-odd), which is time reversal without interchange of initial and final states. Known as the "Collins function", it represents the interference of two amplitudes with different imaginary parts that can account for single-spin asymmetries. Such asymmetries involving longitudinal target polarization have already been observed in pion electroproduction [8]. Theoretical interpretation [9, 10, 11, 12, 13, 14] of those data in terms of transversity-related distributions, as well as theoretical calculations [15, 16] suggest that the Collins function 
has a substantial magnitude, although the effects of pion and gluon rescattering tend to cancel [17]. Thus measurements employing transverse target polarization may be expected to constrain transversity itself. This Letter is the first report of experimental semi-inclusive DIS asymmetries with transverse target polarization.

A completely different possible mechanism for producing target-related single-spin asymmetries has also been identified. It was realized over a decade ago that such asymmetries might arise from correlations between the transverse polarization of the target nucleon and the $p_{T}$ of quarks [18, 19, 20]. A vestige of that quark $p_{T}$ surviving both the photo-absorption and the ordinary fragmentation process can be inherited in the transverse momentum component $\vec{P}_{h \perp}$ of the produced hadron, and can thereby influence its azimuthal distribution relative to the target spin axis. The "Sivers distribution function" $f_{1 T}^{\perp}$ describing the correlation of $p_{T}$ with target polarization is related to a forward scattering amplitude involving helicity-flip of only the target nucleon $\left(N^{\Rightarrow} q \rightarrow N^{\Leftarrow} q\right)$, which must therefore involve orbital angular momentum of the unpolarized quark [21, 22]. Recently this idea has found a reformulation touching on fundamental issues in QCD. It was realized that single-spin asymmetries that can be attributed to such $p_{T}$-dependent parton distributions can also be understood in terms of a final-state interaction (FSI) via a soft gluon 21, 23, 24]. This FSI is a leading-order approximation for a gauge link that is necessary to restore color gauge invariance 25]. A key point is that the FSI offers a mechanism for the interference of amplitudes that is associated with the T-odd nature of the Sivers function, which was once believed to forbid its existence. A related chiral-odd partner $h_{1}^{\perp}$ [26] of the chiral-even Sivers function was found to provide an explanation for the substantial $\cos 2 \phi$ dependence observed in Drell-Yan cross sections [27, 28]. The Sivers function itself is predicted to create Drell-Yan single-spin asymmetries [29], but there it is believed to have the opposite sign to its appearance in DIS, due to the fundamental time reversal symmetry of QCD 23]. This prediction of perturbative QCD needs to be tested experimentally.

Single-spin azimuthal asymmetries arising from the Collins and Sivers mechanisms have a common $\sin \phi$ behavior when the target is polarized along the lepton beam axis, as was the case for all previously published singlespin asymmetries for leptoproduction. However, the additional degree of freedom that is the azimuthal angle $\phi_{S}$ of the axis of transverse target polarization results in distinctive signatures: $\sin \left(\phi-\phi_{S}\right)$ for the Sivers mechanism, and $\sin \left(\phi+\phi_{S}\right)$ for the Collins mechanism [26]. Only the Collins mechanism involves the orientation of the lepton scattering plane because it depends on the influence of the quark's polarization on that component of the transverse momentum $k_{T}$ acquired in the fragmentation process by the struck quark that is orthogonal to its transverse polarization, after its spin component in the lepton scattering plane has been flipped by the photoabsorption. In contrast, the Sivers effect arises through the struck quark "remembering" the $p_{T}$ that it had in the target. In either case, the transverse momentum tends to be inherited by a forward hadron that may "contain" this quark. Hence the hadron $P_{h \perp}$ is correlated with $k_{T}$ $\left(p_{T}\right)$ in the case of the Collins (Sivers) effect.

In the analysis reported here, the cross section asymmetry with respect to the target polarization is extracted as a two-dimensional distribution in $\phi$ versus $\phi_{S}$, which is then fitted with a sum of contributions from the above two sinusoidal dependences. This simultaneous extraction of both contributions was shown by detailed Monte Carlo simulations to avoid significant crosscontamination, even when they have very different magnitudes in the context of a limited detector acceptance.

The data reported here were recorded during the 2002 2003 running period of the HeRmes experiment using a transversely nuclear-polarized hydrogen gas target internal to the $E=27.5 \mathrm{GeV}$ HeRA positron storage ring at Desy. The positron beam was unpolarized at this time. The open-ended target cell is fed by an atomicbeam source based on Stern-Gerlach separation [30] with hyperfine transitions. The nuclear polarization of the atoms is flipped at $60 \mathrm{~s}$ time intervals, while both this polarization and the atomic fraction inside the target cell are continuously measured 31]. The average value of the proton polarization $S_{T}$ was $0.78 \pm 0.04$. Tracking corrections were applied for the deflections of the scattered particles caused by the vertical $0.3 \mathrm{~T}$ target holding field, with little effect on the extracted asymmetries.

Scattered positrons and any coincident hadrons are detected by the HeRMES spectrometer [32. Its acceptance spans the scattering angle range $40<\left|\theta_{y}\right|<140 \mathrm{mrad}$ and $\left|\theta_{x}\right|<170 \mathrm{mrad}$. Hence the azimuthal acceptance is segmented, but this was found in Monte Carlo studies to have negligible effect on the Fourier components of interest. Positrons are identified with an efficiency exceeding $98 \%$ and a hadron contamination of less than $1 \%$ using an electromagnetic calorimeter, a transitionradiation detector, a preshower scintillation counter and a Čerenkov detector. Charged pions are identified using a dual-radiator ring-imaging Cerenkov detector [33.

Events were selected subject to the kinematic requirements $W^{2}>10 \mathrm{GeV}^{2}, 0.1<y<0.85$ and $Q^{2}>1 \mathrm{GeV}^{2}$, where $W$ is the invariant mass of the initial photonnucleon system and $y=(P \cdot q) /(P \cdot k)$. Coincident hadrons were accepted if $0.2<z<0.7$ and $\theta_{\gamma^{*} h}>$ $0.02 \mathrm{rad}$, where $z=\left(P \cdot P_{h}\right) /(P \cdot q)$, and $\theta_{\gamma^{*} h}$ is the angle between the directions of the virtual photon and the hadron. All hadrons detected in each event were included - not only the one with largest $z$.

For each produced hadron type $h$, and for each bin in either $x$ or $z$ or for the entire data set, the asymmetry was evaluated in two dimensions $\phi$ and $\phi_{S}$, where $\phi_{S}$ always indicates the spin direction of the $\uparrow$ state. Defining 
$N_{h}^{\uparrow(\downarrow)}\left(\phi, \phi_{S}\right)$ as the semi-inclusive luminosity-normalized yield in that target spin state, the asymmetry is

$$
A_{U T}^{h}\left(\phi, \phi_{S}\right)=\frac{1}{\left|S_{T}\right|} \frac{\left(N_{h}^{\uparrow}\left(\phi, \phi_{S}\right)-N_{h}^{\downarrow}\left(\phi, \phi_{S}\right)\right)}{\left(N_{h}^{\uparrow}\left(\phi, \phi_{S}\right)+N_{h}^{\downarrow}\left(\phi, \phi_{S}\right)\right)},
$$

The Collins azimuthal moment $\left\langle\sin \left(\phi+\phi_{S}\right)\right\rangle_{U T}^{h}$ and Sivers moment $\left\langle\sin \left(\phi-\phi_{S}\right)\right\rangle_{U T}^{h}$ of the virtual-photon asymmetry are extracted in the fit

$$
\begin{aligned}
\frac{A_{U T}^{h}\left(\phi, \phi_{S}\right)}{2} & =\left\langle\sin \left(\phi+\phi_{S}\right)\right\rangle_{U T}^{h} \frac{B(\langle y\rangle)}{A(\langle x\rangle,\langle y\rangle)} \sin \left(\phi+\phi_{S}\right) \\
& +\left\langle\sin \left(\phi-\phi_{S}\right)\right\rangle_{U T}^{h} \sin \left(\phi-\phi_{S}\right)
\end{aligned}
$$

Here $B(y) \equiv(1-y), A(x, y) \equiv \frac{y^{2}}{2}+(1-y)(1+$ $R(x, y)) /\left(1+\gamma(x, y)^{2}\right), R(x, y)$ is the ratio of longitudinal to transverse DIS cross sections, $\gamma(x, y)^{2} \equiv 2 M_{p} x /(E y)$. The values for $R(\langle x\rangle,\langle y\rangle)$ 34] cannot be neglected here as they fall in the range $0.1-0.34$. The reduced- $\chi^{2}$ values for the fits are in the range $0.74-1.89$. The statistical correlations between the Sivers and Collins moments fall in the range -0.5 to -0.6 . The addition of terms for $\sin \left(3 \phi-\phi_{S}\right), \sin \phi_{S}$ and $\sin \left(2 \phi-\phi_{S}\right)$ resulted in coefficients that are negligible compared to their uncertainties, and in negligible changes to the Collins and Sivers moments. Effects of acceptance, instrumental smearing and QED radiation were all found to be negligible in Monte Carlo simulations 35. The largest contribution to the systematic uncertainties is due to the target polarization.

When the azimuthal moments are averaged over the experimental acceptance, the selected ranges in $x$ and $z$ are $0.023<x<0.4$ and $0.2<z<0.7$, and the corresponding mean values of the kinematic parameters are $\langle x\rangle=0.09,\langle y\rangle=0.54,\left\langle Q^{2}\right\rangle=2.41 \mathrm{GeV}^{2},\langle z\rangle=0.36$ and $\left\langle P_{\pi \perp}\right\rangle=0.41 \mathrm{GeV}$. The dependences of the charged pion moments on $x$ and $z$ are shown in Fig. 2 Also shown are simulations based on Pythia6 [36], tuned for HeRMES kinematics, of the fractions of the semi-inclusive pion yield from exclusive production of vector mesons, the asymmetries of which are poorly determined.

The averaged Collins moment for $\pi^{+}$is positive at $0.021 \pm 0.007$ (stat), while it is negative at $-0.038 \pm$ 0.008 (stat) for $\pi^{-}$. Such a difference is expected if the transversity densities resemble the helicity densities to the extent that $\delta u$ is positive and $\delta d$ is negative and smaller in magnitude, as models predict [37]. However, the magnitude of the negative $\pi^{-}$moment appears to be at least as large as that for $\pi^{+}$. The left panel shows that this trend becomes more apparent as the magnitudes of these transverse moments increase at larger $x$ where valence quarks tend to dominate, as did the previously measured longitudinal asymmetries. However, the large negative $\pi^{-}$moments might be considered unexpected as neither quark flavor dominates $\pi^{-}$production like the

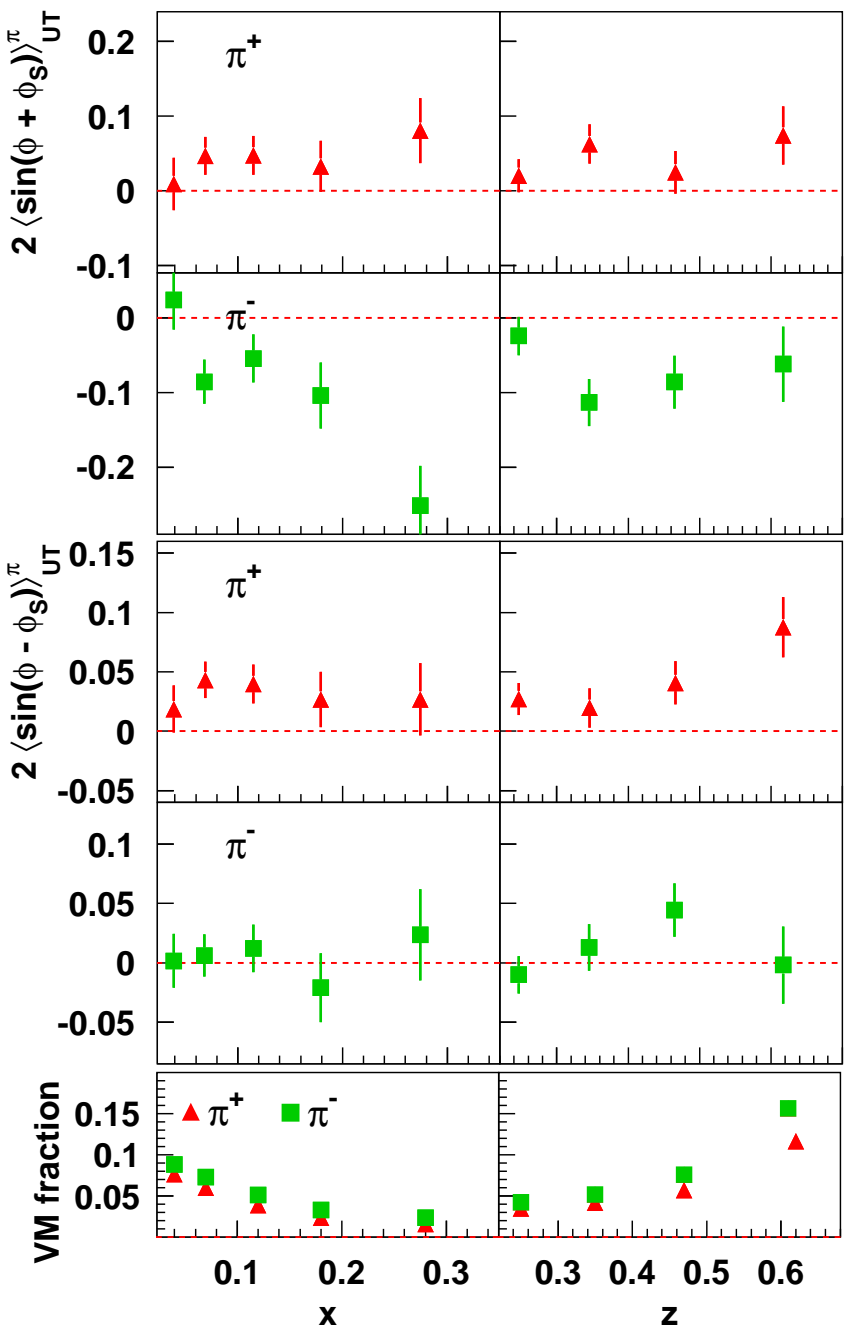

FIG. 2: Virtual-photon Collins (Sivers) moments for charged pions as labelled in the upper (middle) panel, as a function of $x$ and $z$, multiplied by two to have the possible range \pm 1 . The error bars represent the statistical uncertainties. In addition, there is a common $8 \%$ scale uncertainty in the moments. The lower panel shows the relative contributions to the data from simulated exclusive vector meson production.

up quark dominates $\pi^{+}$, and one expects $|\delta d|<|\delta u|$ in analogy with $|\Delta d|<|\Delta u|$. This expectation is reflected in model predictions 13, 14] based on the interpretation of those longitudinal asymmetries. This failure of those predictions could be due to the neglect of T-odd distributions such as the Sivers function, the contribution of sea quarks or disfavored Collins fragmentation.

One explanation of the larger negative $\pi^{-}$azimuthal moments could be a substantial magnitude with opposite sign for the disfavored Collins function describing e.g. the fragmentation of up quarks to $\pi^{-}$mesons. Opposite signs of the favored and disfavored Collins functions might be understood in the light of the string model of fragmentation. If a favored pion forms as the string end created 
by the first break, a disfavored pion from the next break will inherit transverse momentum from the first break in the opposite direction from that acquired by the first pion. Such a $P_{\pi \perp}$ anticorrelation between favored and disfavored pions is demonstrated by the JETSET simulation [36], which is based on a string fragmentation model. Hence any correlation between $P_{\pi \perp}$ and another kinematic or spin observable should have the opposite sign for favored and disfavored pions.

The averaged Sivers moment is positive and nonzero at $0.017 \pm 0.004$ (stat) for $\pi^{+}$, appearing to provide the first evidence in leptoproduction for a T-odd parton distribution function. The $\pi^{-}$moment is consistent with zero: $0.002 \pm 0.005$ (stat). Since the $\pi^{+}$moment is dominated by up quarks, a positive value with the definition of azimuthal angles used here would imply a negative value for the Sivers function of this flavor. Continuing studies of the small sample of exclusive $\rho^{0}$ events in which both decay pions are detected suggest that this asymmetry extracted for the $\pi^{+}$exactly as in the semi-inclusive DIS analysis also has a significant positive tendency. (The Collins asymmetries from this event sample are consistent with zero.) Therefore the Sivers asymmetry from the entire data set must be interpreted with caution.

In summary, a measurement with transverse target polarization of single-spin asymmetries for semi-inclusive electroproduction of charged pions in deep-inelastic scattering has for the first time disentangled two different phenomena that were indistinguishable in previous data. Their signals were extracted as distinctive Fourier components of the dependence of the target-spin asymmetry on the azimuthal angles of both the pion and the target spin axis about the virtual photon direction and relative to the lepton scattering plane. One signal can arise from the transverse polarization of quarks in the target, revealed by its influence on the fragmentation of the struck quark. A surprising feature of the data can be explained by the hypothesis that fragmentation that is disfavored in terms of quark flavor has an unexpected importance, and enters with a sign opposite to that of the favored case. The other signal can arise from a correlation between the transverse polarization of the target nucleon and the intrinsic transverse momentum of quarks, and could provide another indication for nonzero orbital angular momentum of quarks in the nucleon. A significant positive $\pi^{+}$asymmetry has been observed, corresponding to a negative value of the naively T-odd Sivers distribution function describing this correlation, but it may be due to a much larger apparent $\pi^{+}$asymmetry in a small contamination from exclusive production of $\rho^{0}$ mesons.

We gratefully acknowledge the DESY management for its support and the staff at DESY and the collaborating institutions for their significant effort, and our funding agencies for financial support.

[1] A. D. Martin et al., Eur. Phys. J. C 23, 73 (2002).
[2] J. Pumplin et al., JHEP 07, 012 (2002), hep-ph/0201195.

[3] B. Lampe and E. Reya, Phys. Rept. 332, 1 (2000).

[4] J. P. Ralston and D. E. Soper, Nucl. Phys. B152, 109 (1979).

[5] X. Artru and M. Mekhfi, Z. Phys. C 45, 669 (1990).

[6] R. L. Jaffe and X. Ji, Nucl. Phys. B375, 527 (1992).

[7] J. C. Collins, Nucl. Phys. B396, 161 (1993).

[8] A. Airapetian et al. (Hermes), Phys. Rev. Lett. 84, 4047 (2002); Phys. Rev. D 64, 097101 (2001); Phys. Lett. B562, 182 (2003).

[9] M. Boglione and P. J. Mulders, Phys. Lett. B478, 114 (2000).

[10] M. Anselmino and F. Murgia, Phys. Lett. B483, 74 (2000).

[11] A. V. Efremov, K. Goeke, and P. Schweitzer, Eur. Phys. J. C 24, 407 (2002).

[12] B.-Q. Ma, I. Schmidt, and J.-J. Yang, Phys. Rev. D 66, 094001 (2002).

[13] A. V. Efremov, K. Goeke, and P. Schweitzer, Eur. Phys. J. C 32, 337 (2003).

[14] P. Schweitzer and A. Bacchetta, Nucl. Phys. A732, 106 (2004).

[15] A. Bacchetta, R. Kundu, A. Metz, and P. J. Mulders, Phys. Rev. D65, 094021 (2002).

[16] L. P. Gamberg, G. R. Goldstein, and K. A. Oganessyan, Phys. Rev. D 68, 051501 (2003).

[17] A. Bacchetta, A. Metz, and J.-J. Yang, Phys. Lett. B574, 225 (2003).

[18] T. Meng, J. Pan, Q. Xie, and W. Zhu, Phys. Rev. D 40, 769 (1989).

[19] D. W. Sivers, Phys. Rev. D 41, 83 (1990).

[20] M. Anselmino, M. Boglione, and F. Murgia, Phys. Rev. D 60, 054027 (1999).

[21] S. J. Brodsky, D. S. Hwang, and I. Schmidt, Phys. Lett. B530, 99 (2002).

[22] M. Burkardt, Phys. Rev. D 69, 057501 (2004).

[23] J. C. Collins, Phys. Lett. B536, 43 (2002).

[24] X. Ji and F. Yuan, Phys. Lett. B543, 66 (2002).

[25] A. V. Belitsky, X. Ji, and F. Yuan, Nucl. Phys. B656, 165 (2003).

[26] D. Boer and P. J. Mulders, Phys. Rev. D 57, 5780 (1998).

[27] D. Boer, Phys. Rev. D 60, 014012 (1999).

[28] D. Boer, S. J. Brodsky, and D. S. Hwang, Phys. Rev. D 67, 054003 (2003).

[29] M. Anselmino, U. D'Alesio, and F. Murgia, Phys. Rev. D 67, 074010 (2003).

[30] F. Stock et al., Nucl. Inst. \& Meth. A 343, 334 (1994).

[31] C. Baumgarten et al., Nucl. Inst. \& Meth. A 482, 606 (2002); Nucl. Inst. \& Meth. A 496, 263 (2003).

[32] K. Ackerstaff et al. (Hermes), Nucl. Inst. \& Meth. A 417, 230 (1998).

[33] N. Akopov et al., Nucl. Inst. \& Meth. A 479, 511 (2002).

[34] L. W. Whitlow et al., Phys. Lett. B250, 193 (1990).

[35] I. Akushevich et al. (1998), hep-ph/9906408.

[36] T. Sjöstrand et al., Comput. Phys. Commun. 135, 238 (2001).

[37] V. Barone, A. Drago, and P. G. Ratcliffe, Phys. Rept. 359, 1 (2002). 